\title{
Pde-5 inhibitors: are they enough for erectile dysfunction?
}

\author{
Piyush desai ${ }^{1^{*}}$ \\ Director at Desai Diabetes and Endocrine Clinic, Honorary Endocrinologist at Govt. Medical College, Surat, Gujarat, India
}

*Corresponding Author: Piyush desai

Email: drpiyushdesai@gmail.com

\begin{abstract}
Erectile dysfunction is a part of broader sexual dysfunction which also includes decreases libido and premature ejaculation. The Massachusetts Male Aging Study (MMAS) found that 52\% of men between 40 and 70 years old reported having some form of Erectile Dysfunction (ED). ${ }^{1}$ Medical conditions, such as hypertension, diabetes mellitus, and Cardiovascular Disease (CVD), and psychological conditions, such as depression and anxiety, also contribute to sexual dysfunction in middle-aged elderly men. Neurological disorders such as cerebral diseases, spinal cord injuries and peripheral neuropathies contribute to the ED in some patients. Hormonal disorders such as hypogonadism, vascular disorders such as atherosclerosis and peripheral vascular diseases also causes ED. Drugs implicated as a cause of ED include hydrochlorothiazides and beta-blocking agents
\end{abstract}

Keywords: Administered in the context of an IRB-approved experimental clinical research protocol.

\section{Introduction}

Erectile dysfunction (ED) is defined as the inability to achieve a full erection or the inability to maintain an erection adequate for sexual intimacy. Erectile dysfunction is a part of broader sexual dysfunction which also includes decreases libido and premature ejaculation. The Massachusetts Male Aging Study (MMAS) found that 52\% of men between 40 and 70 years old reported having some form of erectile dysfunction (ED). ${ }^{1}$ Medical conditions, such as hypertension, diabetes mellitus, and cardiovascular disease (CVD), and psychological conditions, such as depression and anxiety, also contribute to sexual dysfunction in middle-aged elderly men. Neurological disorders such as cerebral diseases, spinal cord injuries and peripheral neuropathies contribute to the ED in some patients. Hormonal disorders such as hypogonadism, vascular disorders such as atherosclerosis and peripheral vascular diseases also causes ED. Drugs implicated as a cause of ED include hydrochlorothiazides and beta-blocking agents. Medications used to treat depression, particularly the SSRIs such as citalopram, escitalopram, fluoxetine, fluvoxamine, paroxetine and sertraline, may also contribute to ED. Men who are treated with radical prostatectomy, radiation therapy and leutinising hormone agonist/ antagonists for prostate cancer are prone to ED during the course of treatment. Table1 summarizes the causes of ED and Figure 1 depicts a common flowchart for management of ED. $^{2}$

\section{Treatment options for erectile dysfunction}

Treatment begins with proper clinical evaluation which includes psychosexual evaluation and clinical examination. The treatment is directed towards specific findings; however some guidelines have been put in place by American Urology Association (AUA). ${ }^{3}$

Clinical questionnaires have been devised or proper objective evaluation of ED, one of the widely accepted is 'the Sexual Health Inventory for Men' also known as
SHIM. ${ }^{4}$ The SHIM is comprised of five questions scored from 1 to 5; total scores of 22-25 are interpreted as no ED, 17-21 as mild ED, 12-16 as mild-to-moderate ED, 8-11 as moderate ED, and 5-7 as severe ED. Other useful questionnaire is Erection Hardness Score (EHS) ${ }^{5}$, the EHS is a single-item instrument that asks men to rate erection hardness on a scale that ranges from 0 (penis does not enlarge) to 4 (penis is completely hard and fully rigid). For specialty practices when the presenting issue is ED, a more detailed instrument such as the full form of the International Index of Erectile Function (IIEF) may be more useful. ${ }^{6,7}$

\section{Behavior modification and psychosexual therapy}

Life style modification is considered to be the first line management in ED. Men should be encouraged to make necessary changes to benefit their overall health. Men should be informed that ED could be an early sign of underlying cardiovascular disease. Referral to a psychosexual health professional should be considered to promote treatment adherence, reduce performance anxiety, and integrate treatments into a sexual relationship.

\section{PDE-5 Inhibitors}

Sildenafil and other similar analogs (tadalafil, vardenafil, and avanafil) have been recommended by US-FDA for treatment of ED. Their benefits and risks should be discussed in detail with the patients. The mechanism of action for all commercially available PDE5 inhibitors is similar. PDE5 inhibitors inhibit the phosphodiesterase type 5 enzyme from breaking down cyclic guanasine monophosphate (cGMP). This inhibition results in an increase in the concentration of penile cavernosal cGMP that then causes smooth muscle relaxation in the corpus cavernosum vasculature resulting in increased erection hardness and duration in men with ED who have sufficient intact vasculature.

The dose response effects of PDE5 inhibitors are small and nonlinear, which in simple terms means that doubling the 
dose does not double the effect; higher dose may produce higher effects though. It is advisable to start with minimum dose which produces maximum effect. For men with diabetes, sildenafil, tadalafil, and vardenafil appear equally effective.

\section{Adverse effects of PDE5 inhibitors}

The most frequently reported adverse effects were dyspepsia, headache, flushing, back pain, nasal congestion, myalgia, visual disturbance, and dizziness. Daily dosing (which allows men to take a lower dose) was associated with lower rates of frequently-reported adverse effects particularly for headaches compared to on-demand use, which requires a higher dose. Literature suggests that men post- resection of prostrate and men post- radio therapy reported substantially higher rates of adverse effects than did men in the general ED population. Other rare but serious adverse effects are Non-arteritic anterior ischemic optic neuropathy, Skin cancers particularly malignant melanoma, and recurrence of prostate cancer.

\section{Testosterone replacement therapy}

Patient with ED who during evaluation are diagnosed to have low testosterone levels $(300 \mathrm{ng} / \mathrm{dL})$ are benefitted by testosterone replacement therapy. Testosterone deprivation has been shown to result in impairment of Nitric Oxide (NO) synthase release, altered PDE5 expression and activity, impaired cavernosal nerve function, and contribution to veno-occlusive disease in the penis. ${ }^{8}$ It is recommended by both European and American study groups that achieving normal testosterone level is advisable for proper dose optimization and improved efficacy of PDE5 inhibitors. Since testosterone replacement therapy is relatively safe, and men can potentially see an improvement in erectile function, it seems prudent to consider its use for treatment when presented with a patient suffering from ED; however men should be advices that testosterone monotherapy is not very effective as compared to combined therapy with PDE5 inhibitor. ${ }^{9}$

\section{Vacuum erection devices}

Men with ED should be informed regarding the treatment option of a vacuum erection device. Vacuum erection devices are clear plastic chambers placed over the penis, tightened against the lower abdomen with a mechanism to create a vacuum inside the chamber. This directs blood into the penis. If an adequate erection occurs inside the chamber, the patient slips a small constriction band off the end of the vacuum erection device and onto the base of the penis. An erection beyond $30 \mathrm{~min}$ is not recommended. These devices can be a bit cumbersome, but are very safe. Vacuum devices are associated with high rates of patient and partner satisfaction and are an effective and low-cost treatment option for select men with ED. They are effective in the general ED population as well as in men with diabetes, spinal cord injury and post-prostatectomy. In men who are PDE5 inhibitor non-responders, vacuum erection device may have a role as a "rescue" device.

\section{Intra cavernosal injection}

PDE5 inhibitors are ineffective in about $40 \%$ of men. In addition, a significant proportion of men initially responsive to PDE5 inhibitor eventually will become non-responsive as ED progresses and will require a different ED treatment approach. Men who have contraindications to the use of PDE5 inhibitor, men who prefer not to take an oral medication, or men who find that PDE5 inhibitor are inadequate or ineffective may choose the intra cavernosal injection approach to treating ED. Intra cavernosal injections are administered by injecting the medication into the corpus cavernosal of the penis to produce an erection. Some commonly used medications in clinical practice are alprostadil (prostaglandin E1), papavarine, phentolamine and atropine. Combination of medications is also used. Only alprostadil is US-FDA. For men with ED who are considering intra cavernosal injection therapy, an in-office injection test should be performed. The most serious adverse effect associated with intra cavernosal injection medications is priapism. Pain is a common occurrence of intra cavernosal injections; penile fibrosis, plaque and penile deformities have also been reported.

\section{Intra urethral suppository}

Intra urethral medication involves the insertion of a delivery catheter into the meatus and depositing an alprostadil pellet in the urethra to induce an erection sufficient for intercourse. Alprostadil is a treatment option for men for whom PDE5 inhibitor are contraindicated and patients who do not prefer injections. Intra urethral alprostadil should not be prescribed until a man has undergone instruction in the method, an initial dose-titration in the office, and detailed counseling regarding possible adverse effects and actions to take in response to potentially serious adverse effects. Alprostadil is available in doses of $100 \mu \mathrm{g}, 250 \mu \mathrm{g}, 500 \mu \mathrm{g}$, and $1,000 \mu \mathrm{g}$. The clinician should select a dose for in-office testing that is expected to produce an erection sufficient for intercourse, the lowest dose expected to be effective should be used.

\section{Surgical prosthesis}

Another choice for the man with ED is the surgical implantation of a penile prosthesis. The partners should be counseled regarding the benefits and potential risks. The patient should understand that there are several devices available, including malleable (non-inflatable) models as well as two or three piece inflatable prosthesis. The benefits include the ability to generate an erection sufficient for intercourse on-demand, for as long as is desired, and as frequently as desired. The potential risks and burdens of prosthesis surgery include the risks inherent in the surgical procedure, possible changes in the appearance of the penis, and the potential for device malfunction or failure. Men should understand that this treatment choice is best conceptualized as irreversible; although prostheses can be removed, it is unlikely that a man's penis will be reliably responsive to other ED therapies after removal of the prosthesis. Penile prosthetic surgery should not be 
performed in the presence of systemic, cutaneous, or urinary tract infection.

\section{Vascular surgery}

Penile arterial reconstruction surgery may be considered for the man with ED who is young and who does not have veno-occlusive dysfunction or any evidence of generalized vascular disease or other comorbidities that could compromise vascular integrity. Data from most studies indicate that predicting whether reconstructive surgery will result in long-term success for a given man is extremely difficult, even in men without co-morbidities and with good vascular health. In addition, proper diagnosis requires a thorough investigation. A recent study reported that nearly $50 \%$ of men initially identified as good candidates for reconstruction were not properly diagnosed. ${ }^{10}$ For men with $\mathrm{ED}$, penile venous surgery is not recommended.

\section{Future treatment options of ED}

\section{Penile low intensity shock wave therapy}

This is a novel therapy for ED, in this therapy low intensity shock waves are given twice a week for 3 to 6 weeks; this is suggested to cause neo-vascularisation of the cavernosal tissue. The use of low-intensity shock wave lithotripsy may convert PDE5 inhibitor non-responders to responders. ${ }^{11}$ The treatment's ability to restore normal erectile function remains by this therapy remains a question, the duration of treatment effects beyond possible short-term efficacy is not well-established, and the burdens associated with obtaining the treatment (i.e., time and cost) are substantial. Olsen et al. (2014) reported that at the end of the five-week treatment, $57 \%$ of men in the low intensity shock wave group $(n=51)$ had an EHS score of 3 or 4 compared to $9 \%$ in the sham group $(n=54)$.

\section{Platelets rich plasma infusion}

Some reports have been published to document the role of platelet rich plasma (PRP) infusion as a therapy for ED, the
American urology association recommends PRP should not be offered to men with ED unless it is administered in the context of an IRB-approved experimental clinical research protocol. At this time, no full-text peer-reviewed publications are available to constitute an evidence base.

\section{Stem Cells}

Findings from studies that have evaluated ICI stem cell therapy do not indicate that benefits reliably outweigh risks/burdens for men with ED. Neither the most effective source and dose of stem cells nor the duration of treatment effects has been established. Given the paucity of data obtained in human participants, the risks of treatment also are not well-established.

\section{Gene therapy}

One futuristic therapy is gene therapy, that would deliver genes that produce products or proteins that may not be functioning properly in the penile tissue of men with ED. Replacement of these proteins may result in improvement in erectile function. Experimental animal models have demonstrated improvement in erectile function with gene therapy. Human studies may also demonstrate success with this therapy. Gene therapy may take a long time for regulatory approval and public acceptance.

\section{Conclusion}

Erectile dysfunction is a multi-dimensional problem and needs a multi-disciplinary approach for proper evaluation and treatment. PDE-5 inhibitors are mainstay of the treatment, but, other treatment modalities are equally important adjunct. The supportive measures such as psychosexual evaluation and individualized approach are recommended.

Table 1: Causes of Erectile Dysfunction

\begin{tabular}{|l|l|}
\hline \multicolumn{1}{|c|}{ Aging } & \multicolumn{1}{c|}{ Causes of Erectile Dysfunction } \\
\hline Psychological & Depression, anxiety \\
\hline Neurological & Cerebral diseases, spinal cord injury, spinal disease, peripheral neuropathy, pudendal nerve injury \\
\hline $\begin{array}{l}\text { Hormonal disorders } \\
\text { (depresses libido) }\end{array}$ & $\begin{array}{l}\text { Hypogonadism, hyperprolactinemia, hyper- or hypothyroidism, Cushing's syndrome, Addison's } \\
\text { disease }\end{array}$ \\
\hline Vascular disorders & $\begin{array}{l}\text { Atherosclerosis, ischemic heart disease, peripheral vascular disease, venous incompetence, } \\
\text { Cavernosal disorders }\end{array}$ \\
\hline Medications & $\begin{array}{l}\text { Antihypertensives, antidepressants (depresses libido), estrogens and antiandrogens (depresses } \\
\text { libio), digoxin }\end{array}$ \\
\hline Habits & Marijuana use, alcohol abuse, narcotics use, cigarette smoking. \\
\hline Other diseases & Diabetes mellitus, renal failure, hyperlipidemia, hypertension, chronic obstructive pulmonary \\
\hline
\end{tabular}




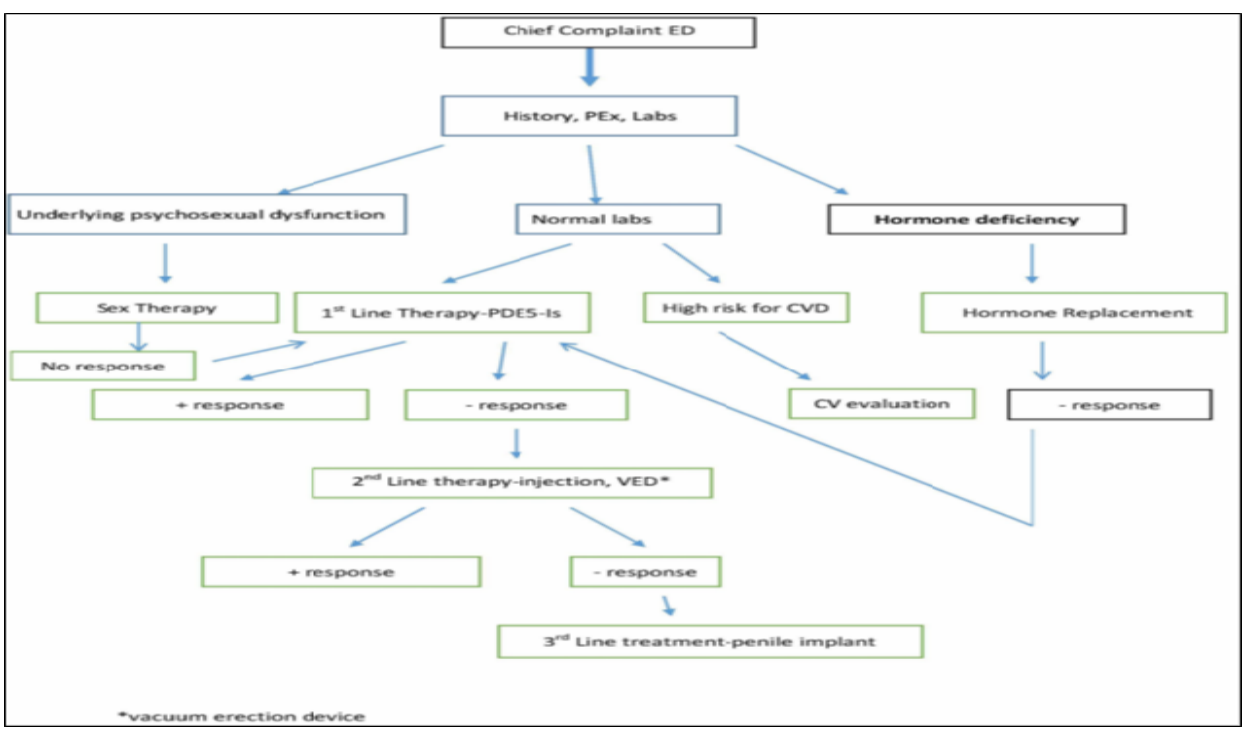

Fig. 1: Flow chart for management of ED

\section{References}

1. Feldman HA, Goldstein I, Hatzichristou DG. Impotence and its medical and psychosocial correlates: results of the Massachusetts Male Aging Study. J Urol 1994;151:54-61.

2. Mobley DF, Khera M, Baum N. Postgrad Med J 2017;93:67985.

3. Arthur L. Burnett, Ajay Nehra, Rodney H. Breau. Erectile dysfunction: AUA guideline. J Urol. 2018;200(3):633-41. doi: 10.1016/j.juro.2018.05.004. Epub 2018 May 7.

4. Rosen RC, Cappelleri JC, Smith MD. Development and evaluation of an abridged, 5-item version of the International Index of Erectile Function (IIEF-5) as a diagnostic tool for erectile dysfunction. Int J Impot Res 1999;11:319.

5. Mulhall JP, Goldstein I, Bushmakin AG. Validation of the erection hardness score. J Sex Med 2007;4:1626.

6. Rosen RC, Riley A, Wagner G. The International Index of Erectile Function (IIEF): a multidimensional scale for assessment of erectile dysfunction. Urology 1997;49:822.
7. Cappelleri JC, Rosen RC, Smith MD. Diagnostic evaluation of the erectile function domain of the International Index of Erectile Function. Urology 1999;54:346.

8. Khera M. Androgens and erectile function: a case for early androgen use in post prostatectomy hypogonadal men. $J$ Sex Med 2009;6:234-8.

9. Bolona ER, Uraga MV, Haddad RM. Testosterone use in men with sexual dysfunction: a systematic review and meta-analysis of randomized placebo-controlled trials. Mayo Clin Proc 2007;82: 20

10. Dabaja AA, Teloken P and Mulhall JP: A critical analysis of candidacy for penile revascularization. $J$ Sex Med 2014;11:2327.

11. Frey A, Sønksen J, Fode M. Low-intensity extracorporeal shockwave therapy in the treatment of post prostatectomy erectile dysfunction: a pilot study. Scand J Urol 2016;50:1-5.

How to cite this article: Desai P. Pde-5 inhibitors: are they enough for erectile dysfunction?. J Nutr Metab Health Sci 2019;2(4):100-3. 